\title{
Time-lapse Cross-equalization by deep learning
}

\author{
Abdullah Alali ${ }^{1}$, Vladimir Kazei ${ }^{1}$, Basmah Altaf ${ }^{1}$, Xiangliang Zhang ${ }^{1}$, Tariq Alkalifah ${ }^{1}$
}

December 22, 2020

\begin{abstract}
Ideally, time-lapse seismic data from different vintages should be identical except at the target area (i.e., the reservoir). However, it is almost impossible to have identical data because of many factors, such as different positioning of the sources and receivers and near-surface velocity variation, which result in $4 \mathrm{D}$ noise and reduce the repeatability of the data. To increase the 4D signal and reduce the noise, time-lapse cross equalization methods aim to match the monitor data to the baseline. Here, we propose to implement the cross equalization intelligently using deep learning models. We specifically use a convolutional autoencoder trained on the base data to later predict the matching using another fully connected neural network in the latent space. We implement the approach on a synthetic data and show an improvement in the repeatability by imaging the reservoir and computing the normalized root mean square.
\end{abstract}




\section{Introduction}

Time-lapse (TL) seismic, also known as 4D seismic, is the process of repeatedly acquiring seismic data in the same area but at different times. TL plays a significant role in monitoring fluid substitution in a reservoir such as monitoring $\mathrm{CO}_{2}$ injection. The quality of the TL data depends mainly on the repeatable signal (4D signal) relative to the non-repeatable noise (4D noise) (Nguyen et al., 2015). With perfect repeatability, the differences between the baseline and monitor data reflects only the fluid changes in the reservoir. Obtaining good repeatability is very challenging due to ambient noise, sourcereceiver positioning, velocity variation in the near-surface and other survey factors (Bakulin et al., 2012; Shulakova et al., 2014; Nguyen et al., 2015).

Various TL techniques have been proposed to improve the 4D signal. They are generally composed of improving the acquisition and simultaneous 4D pre-stack processing based on the cross-equalization (XEQ) method (Nguyen et al., 2015). In this research, we will focus on the XEQ in TL processing that aims to correct for the small temporal differences between the surveys. The most commonly used XEQ technique is the matched-filtering (Rickett and Lumley, 2001; Robinson and Treitel, 2000). It is implemented by finding a convolutional operator matching the monitor to the baseline data. To avoid over matching that can zero-out the differences caused by the target signals, the operator is found in a short time window such that it does not contain any reservoir signal (Rickett and Lumley, 2001). This setup does not allow us to accommodate variations of the needed correction with time.

In recent years, many deep learning (DL) methods have been proposed for seismic data preprocessing (e.g. Ovcharenko et al., 2017, 2019; Sun and Demanet, 2019) and inversion (Araya-Polo et al., 2018; Sun and Alkhalifah, 2019; Kazei et al., 2019). They are proven to be powerful tools in learning non-linear functions and can help construct better cross-equalization processes. XEQ can be perceived as training a single layer convolutional neural network. Deep networks are known to outperform those in various signal processing tasks. In this abstract, we build a deep learning-based cross-equalization (DLXEQ). Similar to the conventional XEQ, we propose to train the DL network on short shallow windows that do not contain the target signal and then we infer to the deeper parts. This allows the network to learn the static variation needed to match the surveys while preserving the reservoir signal.

\section{Method}

We propose to train two DL models. The first model is a convolutional autoencoder and the second model is a fully connected neural network. The goal for the former is to learn a representation of the data in a reduced dimension since it is easier to match in a smaller space. The latter is a fully-connected neural network model responsible to implement the matching between the monitor and the baseline. In both models, Adam is used as an optimizer with mean square error loss.

\section{1- Convolutional Autoencoder (CAE)}

A convolutional autoencoder is a neural network model that consists of two main parts. The first part (Encoder) reduces the dimensionality of the data to a latent space while the second part (Decoder) reconstructs the original dimension of the data. Courtesy of the convolutional layers, CAE can exploit and capture spatial patterns which makes it preferred over the traditional dimensionality reduction methods such as principal component analysis and discrete cosine transform (Liu and Grana, 2019).

The CAE structure is shown in Figure 1. It takes a 1D input of length 200 samples and reduces the dimension to 50 in the latent space. One could reduce the dimension even more but we found this to be good enough for our example that we will show in the result section. Since the monitor and the baseline are very similar, the input for the autoencoder can be traces from all the surveys. 


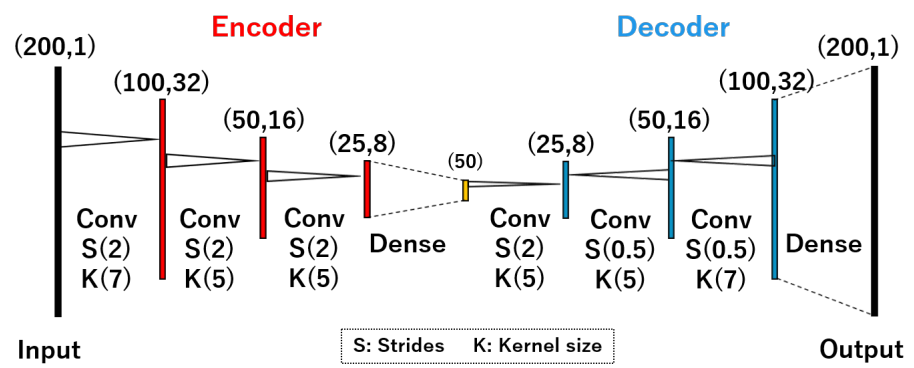

Figure 1 The convolutional autoencoder (CAE) model.

\section{2- Fully connected neural network (FCNN)}

The goal of the FCNN is to map the monitor data in the latent space to the baseline data. Therefore, the input for the FCNN is the encoded samples from the monitor and the output is the encoded samples from the baseline. In our application, we use an FCNN with five hidden layers. The number of units in each layer is four times the original space and the activation function is tanh.

After training, we can assemble the two models into a single one with the form "Encoder-FCNNDecoder" and use it directly for inference in the reservoir region without further training. One could apply a final training to the integrated model but it will only train for a few epochs with negligible loss improvement. It is important to do the training on windows that exclude the reservoir signal to avoid over matching: that is to avoid training the network to zero-out the reservoir signal. A summary of the workflow is shown in Figure 2.
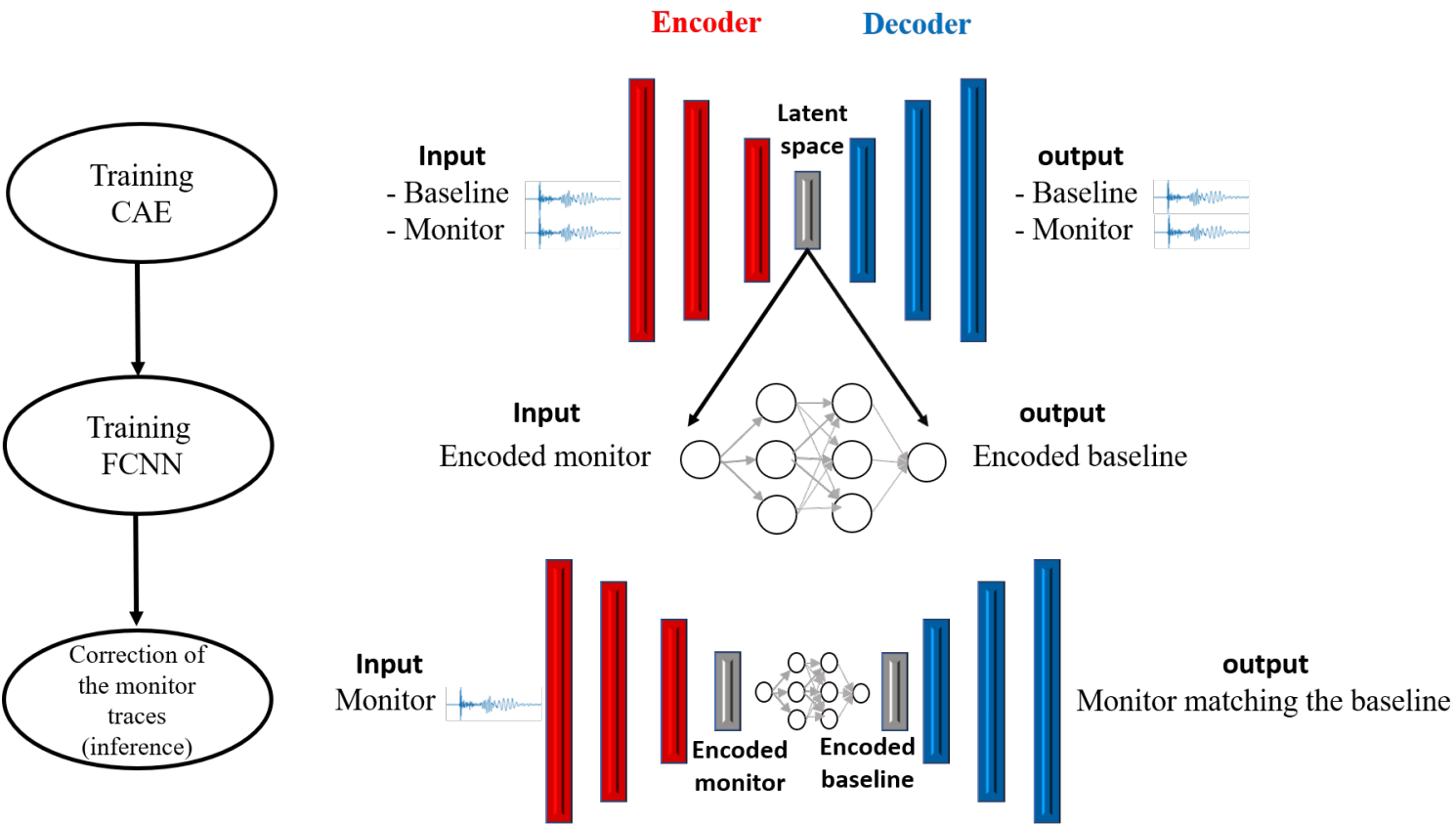

Figure 2 Summary of the workflow.

\section{Training}

To validate the proposed approach, we use the acoustic wave equation to generate synthetic time-lapse data. The Otway velocity model given in Figure 3(a) is used for the baseline. For the monitor, we added random noise to the model with $50 \mathrm{~m} / \mathrm{s}$ mean and $100 \mathrm{~m} / \mathrm{s}$ standard deviation in the first $20 \mathrm{~m}$ depth in addition to the reservoir signal as shown in Figure 3(b). We applied 121 shots separated by $20 \mathrm{~m}$ with a $25 \mathrm{~Hz}$ Ricker wavelet. We record the data for $2.5 \mathrm{~s}$ using 605 receivers. 


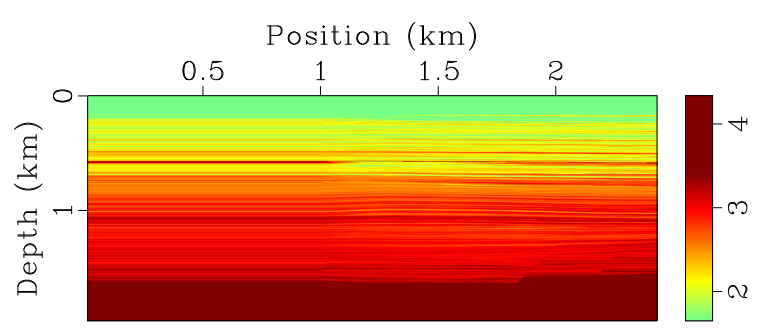

(a)

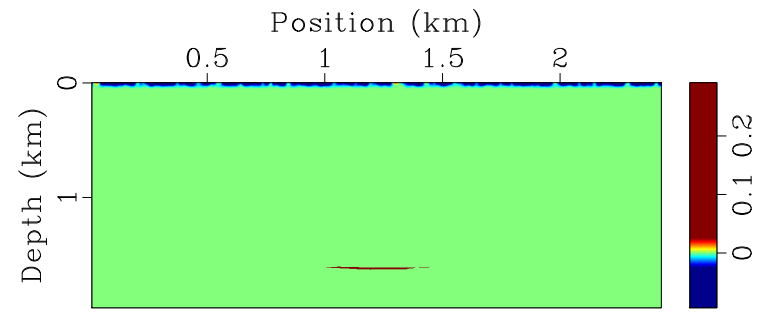

(b)

Figure 3 (a) The Otway velocity model (Glubokovskikh et al., 2016) used to generate data for the baseline and $(b)$ is the reservoir signal and near surface noise added to the baseline for the monitor data.

For training, we choose a time window from $0.4 \mathrm{~s}$ to $1 \mathrm{~s}$ (500 time-samples) as the reservoir signature has not yet been recorded in this window. Since the CAE in Figure 1 accepts an input of length 200 time-samples, we divide the selected time window into batches of size 200 time-samples with 100 timesample overlaps. In the far-offsets, the time-samples are mostly zeros with no signal and that affects the convergence of the training. Therefore, we did not consider the traces that exceed $10 \%$ of the time samples without any signal. We consider $60 \%$ of the data for training and $40 \%$ for validation. The total number of training samples is about 40,000. The data are scaled to be in the range of -1 and 1 for the training. After training the CAE, we use it to encode the selected window of the monitor and the baseline data. The encoded monitor is used as input for the FCNN and the encoded baseline as output. Similar to CAE, $60 \%$ of the data is used for training and $40 \%$ is used for the validation.

\section{Results}

We choose a time-window of 200 time samples from $1.2 \mathrm{~s}$ to $1.6 \mathrm{~s}$ that contains the target. In Figure 4, we show five shot gathers within the selected window for the true reservoir signal, the difference between the baseline and the monitor before the correction and after the correction using DLXEQ, respectively. The reservoir signal clearly improved after applying our approach. Note that similar to the training, we exclude the traces in the far offsets that do not have any signal in the first $10 \%$ time sample and mute them in the following analysis. We then apply reverse time migration to get the reservoir images using the window of shot differences. The true reservoir image (Figure 5(a)) is obtained from the data difference between the baseline and the monitor, excluding the near-surface noise. Figure 5(b) and 5(a) show the image before and after applying the correction, respectively. There is a slight deformation in the reservoir signal in addition to the noise before the correction. After the correction, we can see that most of the noise is removed and the reservoir signal is corrected.

To quantify the improvement, we measure the repeatability by the normalized root mean square (Kragh and Christie, 2002). After applying the DLXEQ, we found that the NRMS is reduced from $35 \%$ to $14 \%$, which suggests that the repeatability has improved.

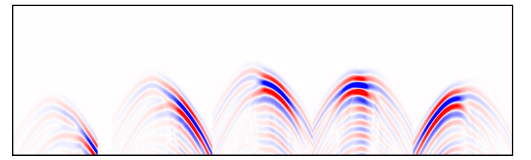

(a)

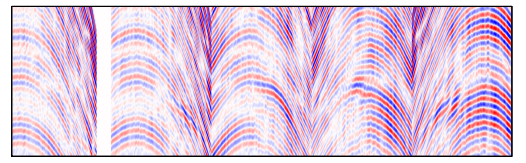

(b)

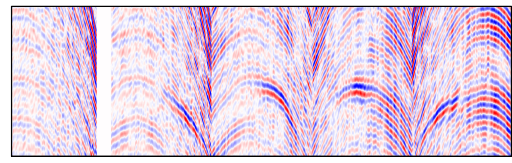

(c)

Figure 4 Shot gathers within 1.2-1.6 $s$ window for (a) the true reservoir signal, $(b)$ baseline - monitor and (c) baseline - monitor after the correction.

\section{Conclusion}

We proposed a new method to apply XEQ between time-lapse data using two DL models: CAE to reduce the dimensionality of the data and FCNN to remove the 4D noise from encoded monitor data. The networks are trained separately. Separating the two models in the training allows for more control and constrain of the FCNN to match the data. We implemented the proposed method on synthetic 


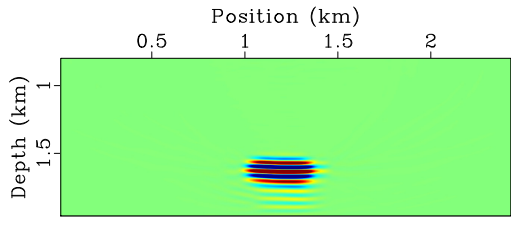

(a)

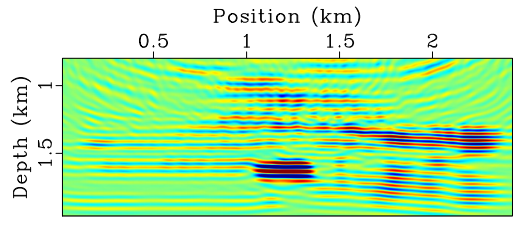

(b)

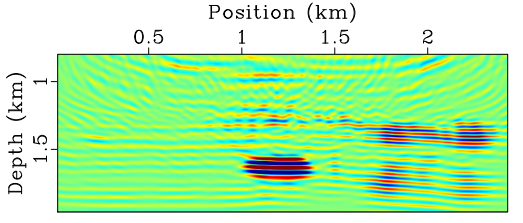

(c)

Figure 5 Images for (a) true reservoir, (b) baseline - monitor and (c) basline - monitor after the correction.

data and showed that the method enhances the repeatability and improves the target signal. The real data is more noisy than the synthetic used in this research. However, a well-known application of the $\mathrm{CAE}$ is noise suppression. This gives the potential of using this approach even with noisy data by only processing at least one or two surveys and train on them.

\section{Acknowledgment}

We would like to extend our appreciation to Saudi Aramco for sponsoring this project especially Philippe Nivlet and Robert Smith for the helpful discussion. We also thank King Abdullah University of Science \& Technology (KAUST) and the members of Seismic Wave Analysis Group (SWAG) for their support.

\section{References}

Araya-Polo, M., Jennings, J., Adler, A. and Dahlke, T. [2018] Deep-learning tomography. The Leading Edge, 37(1), 58-66.

Bakulin, A., Burnstad, R., Jervis, M. and Kelamis, P. [2012] The feasibility of permanent land seismic monitoring with buried geophones and hydrophones. In: 74th EAGE Conference and Exhibition incorporating EUROPEC 2012.

Glubokovskikh, S., Pevzner, R., Dance, T., Caspari, E., Popik, D., Shulakova, V. and Gurevich, B. [2016] Seismic monitoring of CO2 geosequestration: CO2CRC Otway case study using full 4D FDTD approach. International Journal of Greenhouse Gas Control, 49, 201-216.

Kazei, V., Ovcharenko, O., Plotnitskii, P., Peter, D., Zhang, X. and Alkhalifah, T.A. [2019] Mapping full seismic waveforms to vertical velocity profiles by deep learning. submitted to Geophysics.

Kragh, E. and Christie, P. [2002] Seismic repeatability, normalized rms, and predictability. The Leading Edge, 21(7), 640-647.

Liu, M. and Grana, D. [2019] Time-lapse seismic history matching with iterative ensemble smoother and deep convolutional autoencoder. Geophysics, 85(1), 1-63.

Nguyen, P.K., Nam, M.J. and Park, C. [2015] A review on time-lapse seismic data processing and interpretation. Geosciences Journal, 19(2), 375-392.

Ovcharenko, O., Kazei, V., Kalita, M., Peter, D. and Alkhalifah, T.A. [2019] Deep learning for lowfrequency extrapolation from multi-offset seismic data.

Ovcharenko, O., Kazei, V., Peter, D. and Alkhalifah, T. [2017] Neural network based low-frequency data extrapolation. In: 3rd SEG FWI workshop: What are we getting.

Rickett, J. and Lumley, D. [2001] Cross-equalization data processing for time-lapse seismic reservoir monitoring: A case study from the Gulf of Mexico. Geophysics, 66(4), 1015-1025.

Robinson, E.A. and Treitel, S. [2000] Geophysical signal analysis. Society of Exploration Geophysicists.

Shulakova, V., Pevzner, R., Dupuis, J.C., Urosevic, M., Tertyshnikov, K., Lumley, D.E. and Gurevich, B. [2014] Burying receivers for improved time-lapse seismic repeatability: CO2CRC Otway field experiment. Geophysical Prospecting, 63(1), 55-69.

Sun, B. and Alkhalifah, T. [2019] ML-descent: an optimization algorithm for FWI using machine learning. In: SEG Technical Program Expanded Abstracts 2019, Society of Exploration Geophysicists, 2288-2292.

Sun, H. and Demanet, L. [2019] Extrapolated full waveform inversion with deep learning. arXiv preprint arXiv:1909.11536. 